\title{
Network Analysis of Eight Technological Systems.
}

\author{
R. Leoncini and S. Montresor
}

\section{Introduction}

The systemic nature of technological change is now a well established result of much literature, of both theoretical (e.g. Carlsson \& Stankiewicz, 1991; Lundvall, 1992; De Liso \& Metcalfe, 1996; Edquist, 1997) and empirical (e.g. Nelson, 1993; Patel \& Pavitt, 1994; Saxenian, 1994; Carlsson, 1995) nature. As the innovative process does not follow a 'linear', isolated path, but occurs within specific institutional contexts of interactive relationships between different organisations, concepts such as those of innovative and technological systems appear to be the most appropriate units of analysis. However, several specifications have been provided, by referring to both functional and geographical boundaries, which are differently characterised and thus determine differences in the level of analysis and in methodologies.

As far as this paper is concerned, we will adopt a broad and structured notion of technological system that we deem particularly suitable to retain relationships that are not only innovative as such, but also techno-economic - i.e. related not only to the functioning of the innovative and of the production sub-systems, but also of the market (both domestic and foreign) and the institutional infrastructure. footnote Furthermore, our definition refers to national technological systems, because we claim that, even in a world of increasing globalisation and localisms, national boundaries still matter: for example, interesting idiosyncrasies and clusters emerge by looking at the 'configurations' that technological systems assume in different countries (Leoncini \& Montresor, 1998).

This paper aims at measuring and comparing some of the key relationships within a technological system by considering its intersectoral techno-economic linkages. footnote We thus intend to highlight the role of innovative flows of different magnitude within it, the location of its 'cores' and 'terminals', the inward or outward configuration of its partitions, from both a 'cross-sectional' and a 'time series' comparative perspective.

The application refers to eight OECD countries along three temporal spans (see Appendix A1 for full details). It consists of a network analysis of the intersectoral innovation flows matrices we have obtained by disaggregating each technological system into 15 manufacturing sectors (Appendix A2).

The paper is organised as follows. In Section 2 the methodological issues about innovation flows matrices and network analysis are briefly reviewed. Section 3 describes the empirical results. Section 4 contains the main conclusions.

\section{Input-output approach and network analysis}

The approach that we have adopted in this paper mainly follows up a methodology which tries to combine two complementary methods, that is, input-output analysis and network analysis. The basic rationale which underlines the use of input-output analysis in the study of technological systems is briefly restated in Section 2.1. The 'technological' meaning of the most typical network analysis indicators is then discussed in Section 2.2.

The input-output matrix of innovation flows

An input-output approach to the concept of technological system can be developed by combining the input-output table of intermediate (or capital, if available) goods with a conformable matrix of sectoral innovative efforts (R\&D), thus obtaining a particular 'input-output' matrix of innovation flows, $R(n \times n)$. footnote

Since it measures innovative flows that are 'embodied' in the productive flows exchanged among sectors, $R$ can actually account for techno-economic kinds of relationships, which are shaped both by the innovative sub-system (proxied with the R\&D expenditure) and by the 
productive sub-system (proxied with the input-output tables). Furthermore, if, as in the present case, $R$ is obtained by resorting to the notion of vertically integrated sectors, using the Leontief multipliers, the market sub-system is implicitly retained through the final demand vector, while both direct and indirect relationships between sectors can be caught. footnote

As we are particularly interested in comparing technological systems with respect to structural and relational elements, in what follows the matrix $R$ is transformed into a matrix $C(n \times n)$, by considering a matrix of normalised intersectoral innovative acquisitions. In so doing, the matrix obtained is 'relativised', and the scale differences between the sub-systems are neutralised, as well as the intrasectoral flows, which can thus be neglected.

Network analysis

Although mainly developed in economic sociology (Scott, 1991), network analysis indicators and techniques can be applied to examine the structure and the characteristics of a technological system. In the present case, it can be directly performed on the basis of the matrices of innovative flows, $C(n \times n)$, treating the $n$ sectors as 'nodes' and their innovation flows as 'edges'.

In particular, the density and the centralisation of the networks may provide information about the degree of connectivity (Section 2.2.1.), while the centrality degree of the nodes and the oriented graphs (Section 2.2.2.) allow us to map innovative flows of a certain magnitude.

\section{Density}

The density of a network composed by $n$ nodes is generally defined as the ratio between the actual number of edges, $s$, and the maximum number of directed edges:

$$
D=\frac{s}{n(n-1)}, \quad 0<D<1 .
$$

It is straightforward that greater values of ( ref: eq1 ) indicate denser networks. The density of the network corresponding to a technological system can thus be assumed to measure its internal cohesion. That is, the higher is the density of the network, the more connected is the technological system, and vice versa.

In a valued network, such as that of the technological system of the $C$ matrix, the 'actual' number of edges (in this case the number of innovative flows) is usually the number of edges smaller than a certain threshold value $k$, that is $s_{k}$ (Scott, 1991; Faust \& Wasserman, 1995). This amounts to considering dichotomised matrices, $C^{d i c}$, which are binary transformations of the original matrices $C$, made up of $1 s$ and $0 s$ according to a 'greater than' test performed with respect to the 'cut-off' value $k$ :

$$
C_{i j}^{d i c}=1 \text { if } C_{i j}>k ; C_{i j}^{d i c}=0 \text { if } C_{i j} \leq k .
$$

As the choice of an exogenous, usually arbitrary, threshold value for $k$ is one of the main limitations of this indicator, for each technological system we have built up a series of dichotomised matrices by using, rather than a scalar, $k$, a set of cut-off vectors, $\left[\mathbf{k}_{1}, \mathbf{k}_{2}, \ldots, \mathbf{k}_{z}\right]$, each one made up of the ordered distribution of the values of $C$ for country $z$ (in our dataset $z=8$ ). In so doing the choice of the cut-off values is carried out endogenously, on the basis of the characteristics of the investigated sample.

In order to compare the density distributions across different countries, it is helpful to jointly consider their diagrammatic representations: in so doing, it is necessary to select one country $z^{*}$ and to work out the density distributions of all the systems of the sample with respect to $\mathbf{k}_{z^{*}}$. It is immediate that, by measuring relative 'distances', the choice of country $z^{*}$ is not crucial for the final result, but rather for the clarity of the diagrammatic representation.

Centrality, centralisation and oriented graphs

While density is a characteristic of the whole network, centrality is instead proper of each one of its nodes. It measures how central a node is through the number of its connections to and from the other nodes of a network (Freeman, 1979). footnote Formally, the inward (in) and the outward (out) degree centralities of a certain node $j$ are in general defined as follows:

$$
G_{\text {in }}^{j}=\sum i_{\text {in }} ; G_{\text {out }}^{j}=\sum i_{\text {out }}
$$


where $i_{\text {in }}$ and $i_{\text {out }}$ indicate one of the edges which comes, respectively, in and out of node $j$. It is hence immediate that $0<G_{(\cdot)}^{j}<n-1$ and that the greater is $G_{(\cdot)}^{j}$, the more central is the node $j$, either with respect to the incoming edges $\left(G_{i n}^{j}\right)$, or to the outcoming ones $\left(G_{\text {out }}^{j}\right)$, or with respect to both. footnote

Also the centrality indicator assumes a particular meaning when the network considered is that of the technological system (matrix $C$ ). Since the inward and the outward edges stand now for intersectoral innovative acquisitions and diffusions, respectively, the two measures of centrality help in determining whether a sector is pervasive or dependent.

Obviously, in order to perform this analytical examination of the sectoral nodes it is necessary to extract one or more selected cut-off values $k \mathrm{~s}$ from the $\mathbf{k}_{z}$ vectors described above. Thus, ( ref: eq3 ) is evaluated for the innovative acquisitions and diffusions which 'survive' the dichotomisation with respect to $k$. Although the analysis of the density distribution may be of help, the choice of the cut-off value is not free of a certain degree of arbitrariness.

Although it is to be referred to a single sector, the degree centrality can also be used to analyse the nature of the whole technological system, at least in two ways.

First of all, centrality indices can be combined to work out the inward and the outward degree centralisation of one network, defined, respectively, as follows: footnote

$$
H_{\text {in }}(k)=\frac{\sum_{j}\left(G_{\text {in }}^{j_{*}}-G_{\text {in }}^{j}\right)}{(n-1)(n-2)} ; H_{\text {out }}(k)=\frac{\sum_{j}\left(G_{\text {out }}^{j_{*}}-G_{\text {out }}^{j}\right)}{(n-1)(n-2)}
$$

where $G^{j_{*}}$ is the centrality value of the most central node, $j_{*}$, either outward or inward.

According to ( ref: eq4), the centralisation indices measure the 'centrality-gap' between each node and the most central one, relative to the maximum level of centrality of a network composed by $n$ nodes (i.e. $(n-1)(n-2))$. In general, thus, a high index of centralisation identifies a network with wide gaps between the (centrality) positions of the nodes, while a low value identifies a network with similar (centrality) positions. In the present case, this corresponds to technological systems whose sectoral partitions can be deemed, respectively, 'hierarchic'(i.e. high degree centralisation) and 'parithetic' (i.e. low degree centralisation). The former case is less conducive to interactive innovative relationships than the latter.

A second systemwide application of the centrality indices can be obtained by examining their sectoral distributions. In this way it is, for example, possible to identify the composition of a technological system in terms of innovative 'cores' and 'terminals'. Intuitively, we define 'cores' those sectors, or clusters of sectors, that 'count' relatively more in terms of the number of sectors to which they transfer innovation flows (dominance of outward relationships). The contrary holds for the 'terminals', that is the sectors from which innovations are acquired (dominance of inward relationships).

The numerical balance between 'cores' and 'terminals' is an important element in comparing the structure of different technological systems and in relating it to their 'connectivity degree', in turn proxied by their density and centralisation. On the other hand, it is as much important to analyse how the former map into the latter, determining which sectors are innovated by which. This would actually further specify the analysis from a qualitative point of view.

In this last respect, it is convenient to integrate the centrality analysis with that of the directed graphs which correspond to each of the selected dichotomized matrices $C^{d i c}(k)$ : there is indeed a 'bijection' between the set of matrices and the set of directed graphs.

Although the concept of oriented graph and its varieties are defined through rigorous mathematical definitions, footnote in what follows we are only interested in distinguishing different kinds of graphs in qualitative terms. In this respect, it should be noted that as the present analysis is based on vertically integrated sectors, direct and indirect relationships are revealed jointly by a unique innovative flow. For this reason, the directed graph that is associated to a certain dichotomized matrix has to be interpreted as an ensemble of 'innovative couples' (univocal and biunivocal) and (possibly) 'development blocks' (DeBresson, 1996, pp. 167-168). On the contrary, 
standard or non standard 'trees' and 'cycles', or more simple 'technological complexes' do not apply to the same networks, which can be read at most as particular 'cliques' (DeBresson, 1996, pp. 169-171). Although this consideration introduces a fortiori a certain structural homogeneity, relevant differences can however be highlighted across different technological systems in comparing the sectoral composition of the 'innovative couples' and the sectoral location of the eventual 'development blocks'.

\section{The empirical investigation}

The empirical investigation performed in this paper refers to eight OECD countries (i.e. Australia, Canada, Denmark, France, Japan, Italy, The Netherlands and the United Kingdom) along three temporal spans (early 80s, middle 80 s, and early 90s). footnote

The analysis of the density distributions (Section 3.1) is performed with respect to the vector of cut-offs of Canada (i.e. $\mathbf{k}_{\text {Canada }}$ ), so that this distribution is the only one to be perfectly linear, while those of the remaining countries are distributed along it. The choice of Canada has been suggested because it allows us to visualize better than the other the evolution of the density ranking in moving from large to small innovative flows.

For obvious scope constraints, given the relatively large number of countries investigated, and along more than one period, the centrality-centralisation analysis and the exam of the directed graphs (Section 3.2) are carried out with respect to a unique threshold value. In particular, facing a trade-off between 'indistinguishable' (with too many links) and 'trivial' (with too few links) networks, we have chosen a relatively small cut-off value $(k=0.005)$, which amounts to retaining innovative flows of large magnitude.

\section{Density}

As far as the density analysis is concerned, the main results that emerge from the inspection of Figures 3.1, 3.2, and 3.3 can be summarised as follows:

(i) In the first period (Figure 3.1), Japan, Germany and France cluster as the most dense technological systems along the whole cut-offs distribution, hinting the opportunity of greater synergetic benefits. The Japanese system, in turn, reveals greater density values than the other ones, confirming the idiosyncratic nature of the former and the structural similarity of the latter. The model of capitalism seems to be relevant also for an 'intermediate' density cluster made up of two Anglo-Saxon systems (United Kingdom and Canada), with a lower degree of connectivity. Finally, Denmark and The Netherlands (the smallest countries of our sample) have the least dense distributions, suggesting how problems of innovative 'critical mass' might hinder extended interactions.

(ii) The picture for the mid 80s (Figure 3.2) resembles that of the previous one, as far as the most dense distributions are concerned, although the gap between the Japanese and the Rhenish systems narrows. The club of the most connected countries narrows while entering the so-called 'globalisation' phase, in which the systems come to interact more intensively and extensively in the international scenario, with clear consequences for the sustainability of their internal innovative networks. With the exception of The Netherlands, which still lag behind in terms of density, also the less connected technological systems get more aligned. Only with respect to a limited range of intermediate cut-off values, Australia, another small country, lays quite apart from the group of the other, which appears now more homogeneous following the substantial gain of Denmark.

(iii) In the early 90s (Figure 3.3), with the exception of the most and the least dense distributions, the alignments between the investigated technological systems becomes even more apparent, hinting how globalisation tends to reduce the diversities among the countries. Relevant changes occur also at the extremes. On the one hand, Japan loses its domain at the advantage of Germany, although only for intermediate cut-off values, while France keeps staying apart. On the other hand, the range of cut-off values for which Australia lags behind is more narrow than in the second period.

In general terms, the analysis of the density shows two main facts. First of all, the clustering of the investigated technological systems are highly affected by structural elements, such as, for example, the relevant model of capitalism and the country-size. Secondly, the eight countries 
investigated show a rather evident process of 'convergence' footnote in the values of the density along the time, especially in the firsst two periods. While in the third period the set of high density countries shows divergent patterns for some cut-off values. To be sure, the convergence does not appear to be absolute. Although the three most 'dense' countries are more similar in their structure in 1990 then they are in 1980, and the same holds (to a lesser extent) for the 'Anglo-Saxon' countries footnote, a clear lag persists between the two groups. Therefore, it seems likely to interpret this evidence as a process of 'conditional' convergence, i.e. a process of inter-club rather than one of intra-club convergence.

Centrality and centralisation of degree

Although limited to relatively large innovative flows (as already said, the cut-off value is 0.005), the sectoral analysis of centrality (Tables 3.1 to 3.3) confirms some 'stylized facts' and some intuitions one can draw on the particular focus of this study.

Although at different degrees in different technological systems and periods, footnote the most traditional sectors - i.e. food, beverages and tobacco (Sector 1), textiles and leather (Sectors 2), wood and furniture (Sector 3) and paper and printing (Sector 4) - are all dependent, as the 'indegrees' are higher than the correspondent 'outdegrees'. In particular, with the only exception of Japan (and of the German paper sector in the first two periods), the same sectors are 'totally' dependent', as the 'outdegrees' are nil: the 'supplier dominated' nature of these branches appears therefore confirmed (Pavitt, 1984). Another general result concerns the most pervasive sectors of each technological system. With some few exceptions - notably that of Japan footnote - these are actually a 'specialized supplier' sector — metal products (Sector 10) — and a 'science-based' sector - chemical products (Sector 5) — whose technology has a dominant 'material' nature and which are therefore prominent when 'embodied' innovative flows are considered. This interpretation obviously better fits the former sector that, unlike the latter, is actually 'totally' pervasive, i.e. with a nil 'indegree' (still apart from Japan and from Germany in the last period). The same kind of argument also holds for those sectors — such as shipbuilding and repairing (Sector 12) and motorveichles (Sector 13) - whose large scale (Pavitt, 1984) naturally calls for consistent intermediate and capital material inputs: with some relevant exceptions - notably that of the German motorveichles - these sectors are actually 'dependent', and in a total degree (apart from the French motorveichles and the Japanese shipbuilding sectors). A general total dependent nature is also revealed by the professional goods sector (Sector 14), although its innovative acquisitions are also substantially 'disembodied'.

The nature of the remaining sectors is much more time and system specific, as it emerges when the sectoral distributions of the centrality indices are considered along with the centralisation index.

At the outset, this kind of analysis shows how in all periods two clusters of technological systems can be identified (Tables 3.1-3.3). The former, with a core constituted of two main pervasive sectors (Sector 10 and Sector 5), at most encompassing few other slightly pervasive branches, and with a quite 'hierarchic' structure (high outdegree centralisation), is made up of Australia, Canada, Denmark, The Netherlands and the United Kingdom. Another one, whose core of pervasive sectors extends more substantially also to other sectors (in addition to Sector 5 and 10), and whose structure is relatively more 'parithetic' (low outdegree centralisation) comprehends France, Germany, and Japan. This partition resembles pretty well other taxonomies that, mainly focussing on purely innovative and atomistic questions, 'split' the same group of countries with respect to their size, their R\&D intensities, patents scores, educational levels, and so on (see, for example, Nelson, 1993). However, as the present one is traced according to techno-economic and relational aspects, it is not fully consistent with them: once technological systems are considered, the 'myopic' way in which the United Kingdom organise the innovative process (Patel \& Pavitt, 1994) actually becomes more decisive and makes it more similar to the less structured, 'small' systems of innovation (Leoncini \& Montresor, 1998).

However, the two clusters are not homogeneous and completely stable in time, so that a more detailed examination is necessary. 
(i) In the first period (Table 3.1), the 'simple-core' group of technological systems is clearly exemplified by the case of Denmark, with only two pervasive sectors (Sector 5 and 10), whose outward centrality is quite different (in favor of Sector 10) and whose interconnections are not mutual. The Netherlands and the United Kingdom appear instead slightly more 'connected', as they extends the same simple core, still unbalanced towards Sector 10, to the machinery sector (Sector 11), and this could hint to the importance of their specialisation in the non-electrical partition of the same sector. Resource-related questions might instead have a role in explaining the Canadian extension to the coal and petroleum sector (Sector 6). On the other hand, in both cases the extension (in terms of outdegree centrality) is not very big, so that the correspondent outdegree centralisations are nearly the same.

As far as the second cluster is concerned, France and Germany appear to be once again quite similar, as shown by the outdegree centralisation indices. The metal-chemical innovative core is now more balanced towards maximum outward centrality values, and also wider. The machinery sector (Sector 11) is almost as much pervasive as Sector 10 and 5, as well as the 'resource intensive' sectors (Sectors 6-9) are not totally dependent, or even slightly pervasive (e.g. the rubber and plastic products (Sector 7)). Centralisation indexes are substantially lower than those in the previous cluster. A further peculiar feature is identified by the motorveichles sector (Sector 13), nearly pervasive in France and highly pervasive in Germany. At this regard specialisation still matters.

Another confirmation comes from the highly idiosyncratic nature of Japan. Although metal products (Sector 10) are not as much pervasive as in France and Germany, the pervasive core is the widest, as it spans from sector 5 to sector 11, at nearly maximum values. Furthermore, the sectors of the 'terminal' - that is the traditional ones (Sectors 1-4) - are less dependent, with the consequence that the (outdegree) centralisation index is lower than in the previous systems.

(ii) Coming to the second period (Table 3.2), Australia, notably another small and quite isolated systems of innovation (Gregory, 1993), joins the cluster of the most 'polarised' technological systems. The dual pervasive core of Denmark gets more balanced (between Sector 5 and 10) and extends, although at a lesser extent, to machineries (Sector 11). This seems to hint to the fact that the 'systemic evolution' of the Danish (but also of the Swedish) system benefited from an active specialised supplier core, although with a consistent foreign penetration (Edquist \& Lundvall, 1993). In this respect, Denmark 'overcomes' Canada, whose core just substitutes 'energy' (Sector 6) for 'machineries' (Sector 11), and The Netherlands, 'catching-up' with the United Kingdom, where the pervasiveness of machineries (Sector 10) and chemicals (Sector 5) also increase.

In spite of these recombinations, the centralisation level of the former group is however still detached from that of the second group, in turn quite stable. The only relevant variation is actually the centralisation increase for France, in turn due to the out-centrality increase in machineries (Sector 11) and non-finished metals (Sector 9). In spite of this change, and of the slight decrease for Japan and increase for Germany, respectively, in the centrality indeces within the 'resource intensive' partition (Sectors 6-9), the Rhenish-Japanese dichotomy still persists.

(iii) In the third period (Table 3.3), Australia keeps its position of the highest centralised technological system, revealing a configuration that appears structurally polarised around no more than two pervasive sectors, while the remaining are totally dependent. Within the same 'simple-core' cluster, the Danish technological system further increases the pervasivity level of chemicals (Sector 5) and machineries (Sector 5), switching from the most to the least centralised position. Once again, this seems to be a case of conditional catching-up, induced by a structural change towards more synergetic interrelationships. A similar pattern can be identified for the United Kingdom, whose two-sector pervasive core becomes more balanced too. A 'dynamic' sub-cluster, made up of Denmark and United Kingdom, seems therefore identifiable within the former cluster, as opposed to a 'structurally myopic' one, made up of Australia and Canada.

The most remarkable changes of the period are however in the second cluster. Although the centralisation is unchanged, Germany radically changes the distribution of its pervasive core, which narrows to chemicals (Sector 5), metal products (Sector 10), machineries (Sector 11) and 
motorveichles (Sector 13), but now with maximum (or nearly maximum) outward degree centrality. The remaining sectors becomes totally dependent, and this seems to point out to an intensive, rather than an extensive structural change, focusing on and exploiting the externalities of the main sectoral specialisations. A different argument holds for France, whose core composition remains basically the same, but where the inward and the outward centrality degrees change, making the system less centralised and more 'interconnected'. The change of Japan, although less relevant and ininfluent on its idiosyncrasy, is instead in the opposite direction. In spite of the increase of outward centrality in Sector 14 and 15, the combined effect of a lower pervasiveness in metals (Sector 10) and energy products (Sector 6), and of a higher pervasiveness in machineries (Sector 11) and rubber and plastic (Sector 7) determines a slight loss of connectivity (higher outdegree centrality).

\section{Oriented graphs}

At the outset, let us observe how the oriented graphs of the $C_{0,005}^{d i c}$ matrices (Figure 3.4-3.6) make the two clusters discussed above immediately apparent. On the one hand, we have Australia, Canada, Denmark, The Netherlands and the United Kingdom, with a set of relatively few 'innovative couples', exclusively (or nearly exclusively) univocal, and mainly (or solely) based on Sector 5 (chemicals) and 10 (metal products). On the other hand, we have France, Germany and Japan, where the innovative couples are more dense, more frequently biunivocal (but not very much), and also based on sectors other than Sector 5 and 10. As suggested by the centrality analysis, the relationship between pervasive and dependent sectors is quite dichotomic so that within the latter, more systemic, cluster it is not possible to identify 'development blocks' or similarly articulated structures (DeBresson, 1996).

In general terms the two groups show quite distinct sectoral specifications.

In all the technological systems of the former group the metal products sector (Sector 10) extends its innovative diffusions over all the remaining ones. Conversely, the chemical sector (Sector 5) innovates only some of them, namely traditional (e.g. Sectors 2, 3 and 4) and resource intensive (e.g. Sectors 6, 7, 8 and 9) sectors, which are technologically closer to it. The diffusions towards the sectors whose technology is more immaterial are instead in general relatively less frequent and specific to certain countries and periods. The machinery sector (Sector 11), when it comes to integrate the previous core, follows a quite different pattern, as its diffusions generally reach only the scale intensive sectors of the classification (i.e. Sector 12 and 13) and the residual branches (Sector 15). The traditional and/or resource intensive sectors are instead affected in some cases only. The sectoral partitions which refer to Sectors 5 and 11 are quite separated (i.e. the relative sectors overlap only slightly) and the same 'pivotal' sectors do not communicate, although they are both innovated by Sector 10. Therefore, a material and an immaterial technology areas seem to emerge. Extra-core diffusions are very limited, or even absent.

Within the second group of countries, the chemical sector (Sector 5) becomes as pervasive as the metal products one (Sector 10), which in turn has a maximum outward centrality. The exception is Japan, for which Sector 5, and not 10, becomes the most pervasive. The same pattern holds for machineries (Sector 11), which always innovate at least $70 \%$ of the remaining ones. Although the three sectoral partitions now overlap more, a fortiori, the pivotal sectors still do not generally communicate. The resource intensive sectors (Sectors 6-9) increase their importance. Sometimes they join Sector 5 (and eventually 10 and/or 11) in a sort of super-core, while in some other cases they constitute a sort of peripheral core. Extra-core diffusion is less exceptional, if not even normal (as in the case of Japan).

A more precise specification of these regularities, and of their temporal evolution, obviously calls for a period-by-period analysis.

(i) In the early 80s (Figure 3.4), and within the first cluster, The Netherlands and the United Kingdom, unlike Canada and Denmark, show a core which marginally extends also to the machinery sector (Sector 11), as it pervades only the most adjacent branches, those of transport equipments (Sectors 12 and 13). footnote The diffusions of the chemical sector (Sector 5) towards the resource intensive partition encompasses energy products (Sector 6) only in the most 
energy-endowed systems (Canada and The Netherlands). Moreover, in Canada the latter also innovates the former identifying a characteristic biunivocal innovative couple.

Within the second cluster, Japan clearly stands out as the system with the largest core. This is in turn made up of a mostly pervasive 'super-core', centered on basic materials and on their chemical transformation (Sectors 5, 6 and 8), and a 'pheriperic core', made up of synthetic and (non finished) metallic products (Sectors 7 and 9), and of (finished) metal products (Sector 10) and machineries (Sector 11). However, the former (Sectors 7 and 9) are neither linked between them nor with other singular nodes, while the latter (Sectors 10 and 11) exceptionally do not innovate the chemicals (Sector 5) and some other traditional and resource intensive sectors. In France and in Germany the supercore is limited to Sectors 5, 10 and 11, still not totally interlinked, while the resource intensive sectors are much less pervasive than in Japan. Energy products (Sector 6) and rubber and plastics (Sector 7), especially in France, are the only ones remarkably pervasive, concentrating their diffusions towards both traditional and other resource intensive sectors. Exceptionally, some of the Japanese traditional sectors (e.g. Sector 2 and 3, Sectors 3 and 4) constitute biunivocal innovative couples and also reach the residual sector (Sector 15). Another relevant extra-core diffusion is that of German motorveichles (Sector 13), affecting, in addition to Sector 4 and 8, as in France, also traditional sectors, such as Sector 1, 2 and 3.

(ii) Coming to the second period (Figure 3.5), the three-nodes pervasive core (Sectors 5, 10 and 11) becomes dominant in the first group of countries, as Australia is the only one exclusively based on Sector 5 and 10. The chemical diffusions become wider, as they now systematically reach also the energy sector (Sector 6) and all the traditional ones, except for food and beverages (Sector 1). footnote A similar trend can be observed for machineries (Sector 11), in particular in the British and in the Danish systems, where it singularly innovate also some 'upper' sectors (i.e. Sector 2, 4, 6 and 8), showing how the traditional and the resource intensive sectors here benefit from virtuous backward linkages. In The Netherlands the same linkages are instead limited to the 'lower' sectors (i.e. Sectors 12, 13, 14 and 15).

The structure of the technological systems within the second group of countries is identical to the previous period, but its specification is slightly changed. The Japanese energy products sector (Sector 6) switches from the supercore to the periphery, loosing its diffusions to Sectors 11, 13 and 14. The reverse holds for ferrous and non ferrous metals (Sector 9), as does not affect the chemical, and some traditional and resource intensive sector. The machineries sector (Sector 11) is mostly pervasive in France and in Germany. Out of the resource intensive sectors, the pervasivity of rubber and plastic (Sector 7) decreases in Japan, getting disconnected from Sectors 5, 8, 9, 10 and 11, while it increases in France and in Germany. The same holds for the French energy products (Sector 6), while the German one only innovates Sector 1. A similar dicothomy holds for the ferrous and non-ferrous metal products (Sector 9), the diffusions of which are in Germany limited to the residual sector (Sector 15), while in France they are connected to the peripheral core, mainly innovating Sectors 12,13,14 and 15. As far as the extra-core diffusions are concerned, the traditional, biunivocal, innovative couples of Japan, and the univocal, motorveichles based, innovative couples of France and, especially, of Germany are still the most representative.

(iii) In the early 90s (Figure 3.6), the first cluster confirms the dichotomy between Australia and the remaining technological systems. Here, in turn, Canada is clearly distinct from the United Kingdom and Denmark at least in two respects. On the one hand, their chemical sector (Sector 5) gets closer to the role it has in the big-countries cluster (it innovates the most immaterial technology based sectors (Sectors 13, 14, 15, and, in the United Kingdom, also Sectors 11 and 12). On the other hand, a similar argument holds for machineries (Sector 11), which also reach some traditional and resource intensive sectors (Sectors 4, 8, 9, and, in the United Kingdom, also Sectors 2, 6, and 7). The combined effect of this two patterns implies a clear reduction in the dicothomy between the terminal sectors that are innovated, quite evident in the previous periods, especially in the early 80s. In this period, therefore, Denmark and the United Kingdom seem to converge towards the structure of the second cluster.

Here also important changes are observable. The most relevant is, as we already said, that of 
Germany, which degenerates in an enlarged supercore of mostly pervasive and interlocked sectors, made up of chemicals (Sector 5), metal products (Sector 10), machineries (Sector 11) and motorveichles (Sector 13). Also France enlarges the standard '5-10-11-Sector core', with the addition of rubber and plastic (Sector 7). The peripheral core of the resource intensive sectors generally reduces its outdegree centrality. Japan maintains its idiosyncratic structure, and also the sectoral specification of both the supercore and the peripheral core (except for the diminishing pervasive role of rubber and plastic). Small changes only occur in the extra-core diffusions: paper and printing (Sector 4) increases its innovative weight, diffusing, in addition to all the other traditional sectors, also Sectors 8,10 and 15. Moreover, relevant diffusions also emerges in the lower part of the classification, particularly that from professional goods (Sector 14) to shipbuilding (Sector 12).

\section{Conclusions}

In this paper we have carried out an empirical analysis of the innovative flows which constitute the skeleton of the technological systems of eight OECD countries in the last decade. Given the systemic nature of the innovative process, we have opted for an analytical instrument, network analysis, which reveals particularly appropriate for the investigation of complex techno-economic interrelationships.

The density analysis, performed to measure the connectivity of the technological systems, shows that separate clusters emerge according to structural kinds of elements, such as size, technological intensity, model of capitalism, and institutional arrangements. Furthermore, the eight countries investigated show a process of 'conditional convergence', hinting that the globalisation phase they entered in the last decade might have also attenuated differences of structural nature, but only in relative terms.

The centrality analysis mainly confirms other standard sectoral taxonomies. However, the exclusive reference to embodied innovative flows ends up with emphasizing the role of specialised suppliers sectors and of sectors whose technology is basically material.

The centrality distributions and the centralisation analyses also point to quite separated groupings, although not completely homogeneous and stable. A first cluster, with a quite 'hierarchic' structure, is made up of Australia, Canada, Denmark, The Netherlands and the United Kingdom. A second one, whose structure is relatively more 'parithetic' comprehends France, Germany, and Japan.

The analysis of the oriented graphs, mapping the sectoral destinations of the innovative flows, further enhances the differences among and inside the previously defined clusters. In all the technological systems of the former the chemical sector innovates only some of the traditional and resource intensive sectors, while the diffusions towards the immaterial technology sectors are instead relatively less frequent. Within the second cluster, the chemical sector becomes generally as pervasive as the metal products one, with a maximum outward centrality. The same holds for machineries. Unlike the former group, the resource intensive sectors gain remarkable importance, and significant innovative couples, sometimes even biunivocal, can be identified in the more traditional sectors (Japan) and in the motorveichles (Germany).

Although these structural characteristics are more or less persistent along the three periods, two major structural changes can however be identified. The more evident is that of Germany, which by the early 90s radically changes the distribution of its pervasive core, which narrows to four (chemicals, metal products, machineries, and motorveichles), with maximum (or nearly maximum) outward centrality. The remaining sectors turn into totally dependent, and this seems to point out to an intensive, rather than an extensive, structural change, addressed to focus on and exploit the externalities of the main sectoral specialisations. The second substantial change is that of Denmark (and to a lesser extent of the United Kingdom) which in the second, and especially in the third period, gets closer to the structure of the 'Rhenish-Japanese' cluster. This change is mainly due to the pervasive role acquired by the machinery sector, with which the other sectors get more vertically integrated.

bibitem Carlsson B. (ed.) (1995), Technological Systems and Economic Performance: the Case of 
Factory Automation, Kluwer, Dordrecht.

bibitem Carlsson B. and Stankievicz R. (1991), On the nature, functions and composition of technological systems, Journal of Evolutionary Economics, vol. 1, pp. 93-118.

bibitem DeBresson C. (ed.) (1996), Economic Interdependence and Innovative Activity. An Input-Output Analysis, Edward Elgar, Cheltenham.

bibitem De Liso N. and Metcalfe S. (1996), On technological systems and technological paradigms: some recent developments in the understanding of technological change, in Helmstädter E. and Perlman M. (eds.), Behavioral Norms, Technological Progress and Economic Dynamics: Studies in Schumpeterian Economics, University of Michigan Press, Ann Arbor, pp. 71-95.

bibitem Edquist C. (ed.) (1997), Systems of Innovation, Pinter Publishers, London.

bibitem Edquist C. and Lundvall B. A. (1993), Comparing the Danish and Swedish Systems of Innovation, in Nelson, R. (ed.), National Innovation Systems. A Comparative Analysis, Oxford University Press, Oxford, pp. 265-298.

bibitem Faust C. and Wasserman S. (1995), Social Network Analysis, Cambridge University Press, Cambridge.

bibitem Freeman L. C. (1979), Centrality in social networks: conceptual clarification, Social Network, vol. 1.

bibitem Gregory R. (1993), The Australian innovation system, in Nelson R. (ed.), National Innovation Systems. A Comparative Analysis, New York, Oxford University Press, pp. 324-352.

bibitem Harary F. (1969), Graph Theory, Addison-Wesley, London.

bibitem Leoncini R.(1998), The nature of long-run technological change: innovation, evolution and technological systems, Research Policy, vol. 27, pp. 75-93.

bibitem Leoncini R. and Montresor S. (1998), Classifying technological systems: an empirical application to eight OECD countries, Quaderni Dynamis, n. 2/98, IDSE-CNR, Milan.

bibitem Leoncini R., Maggioni M. and Montresor S. (1996), Intersectoral innovation flows and national technological systems: network analysis for comparing Italy and Germany, Research Policy, vol. 25, pp. 415-430.

bibitem Lundvall B.A. (ed.) (1992), National Systems of Innovation. Towards a Theory of Innovation and Interactive Learning, Pinter, London.

bibitem Marengo L. and Sterlacchini A. (1990), Intersectoral technology flows. Methodological aspects and empirical applications, Metroeconomica, vol. 41, pp. 19-39.

bibitem Mitchell J. (1969), The concept and use of social networks, in Mitchell J., Social Networks in Urban Situations, Manchester University Press, Manchester.

bibitem Nelson R. (ed.) (1993), National Innovation Systems. A Comparative Analysis, Oxford University Press, New York.

bibitem Papaconstantinou G., Sakurai N. and Wyckoff A. (1998), Domestic and international product-embodied R\&D diffusion, Research Policy, vol. 27, pp. 301-314.

bibitem Patel P. \& Pavitt K. (1994), The nature and economic importance of national innovation systems, STI Review, vol. 14, pp. 9-32.

bibitem Pavitt K. (1984), Sectoral patterns of technical change: towards a taxonomy and a theory, Research Policy, vol. 13, pp. 343-373.

bibitem Saxenian A. (1994), Regional Advantage. Culture and Competition in Silicon Valley and Route 128, Harvard University Press, Cambridge, Mass.

bibitem Scott J. (1991), Social Network Analysis. A Handbook, MIT Press, Cambridge, Mass. 
appendix

AppendixA1. Sample characteristics

\begin{tabular}{||l|c|c||}
\hline \hline & Input-output tables & R\&D expenditure \\
\hline Australia & 1986,1989 & 1986,1989 \\
\hline Canada & $1981,1986,1990$ & $1981,1986,1990$ \\
\hline Denmark & $1980,1985,1990$ & $1980,1985,1990$ \\
\hline France & $1980,1985,1990$ & $1980,1985,1990$ \\
\hline Germany & $1978,1986,1990$ & $1978,1986,1990$ \\
\hline Japan & $1980,1985,1990$ & $1980,1985,1990$ \\
\hline United Kingdom & $1979,1984,1990$ & $1979,1984,1990$ \\
\hline The Netherlands & 1981,1986 & 1981,1986 \\
\hline Source: & OECD, DSTI (STAN, & $\begin{array}{l}\text { OECD, DSTI (STAN, } \\
\text { ANBERD), } 1994\end{array}$ \\
\hline \hline
\end{tabular}

appendix

\section{Appendix A2. Sectoral disaggregation}

\begin{tabular}{|l|l||}
\hline \hline 1 & Food, beverages and tobacco \\
\hline 2 & Textile, apparel and leather \\
\hline 3 & Wood products and furniture \\
\hline 4 & Paper, paper products, printing and publishing \\
\hline 5 & Chemical industry, drugs and medicines \\
\hline 6 & Energy products \\
\hline 7 & Rubber and plastics products \\
\hline 8 & Non-metallic mineral products \\
\hline 9 & Ferrous and non-ferrous metals \\
\hline 10 & Metal products \\
\hline 11 & Non-electrical machinery and electronics \\
\hline 12 & Shipbuilding and repairing \\
\hline 13 & Motor vehicles and other means of transport \\
\hline 14 & Professional goods \\
\hline 15 & Other manufacturing \\
\hline \hline
\end{tabular}

